\title{
DESIGN AND IMPLEMENTATION OF MICROWAVE PARTS
}

\section{OF AIRBORNE-RADAR-WARNING RECEIVERS}

\author{
Hosny A. El-Motaafy ${ }^{*}$, Mamdouh H. Ibrahim ${ }^{* *}$
}

\begin{abstract}
The radar warning receiver (RWR) is the heart of the self protection system of modern aircraft. The most important receiver structures that can be used as RWR are the crystal video recelver (CVR) and the instantaneous frequency measurement (IFM) receiver.

This paper presents a complete design and implementation of the microwave part of the CVR. This CVR is designed to cover a frequency ranging from 1.5 up to $4 \mathrm{GHz}$. The CVR is implemented using the recently developed microstrip technology. The designed receiver was successfully tested and the results in terms of the output voltage and the voltage standing wave ratio (VSWR) were very good.

This paper presents also a complete design and implementation of the critical components of the IFM receiver. These components are the phase correlator, the power dividers, the delay line, the square law detectors preceded by broadband matching circuits, and the low pass filter. They are implemented using the microstrip technology. The input to the correlator is the threatening radar signal and the output comprises four video signals that can be processed to obtain the signal frequency instantaneously. A new design procedure is proposed to overcome the problems, usually encountered when trying to design and implement phase correlators. The designed correlator was successfully tested and found to operate adequately in the whole S-band.
\end{abstract}

\section{INTRODUCTION}

Radar warning receivers are used in military aircrafts and helicopters to warn of an attack by surface-to-air missiles (SAM), air interceptors (AI), and anti-aircraft gun systems. They are also used to provide warning to tank crews of imminent missile or gun attack, and they are used by submarines on or near the surface to warn when an aircraft's surface search radar or weapon systems is illuminating the submarine. Once alerted to the type, direction, and relative priority of the threats, the crew can take the following possible action $[1-2]$ :

- Pilot/operator maneuvers so as to reach a safer region, or makes evasive maneuver with respect to the weapon systems.

- Passive countermeasures are activated to deceive the tracking radar, if the aircraft is equipped with them.

* Ass. Prof. Dr., Dept. of Electronic Warfare Eng., M.T.C

** Dept. of Radar, M.T.C 
- An active countermeasures system is automatically activated to disable the weapon system.

- If the mission provides for it, an anti-radiation missile is launched.

Since the applications of RWRs are essentially military, the design techniques of these receivers and the technologies used for their implementation are highly classified. Hence, this paper presents a design and an implementation of RWRs the authors did in an effort to master the RWR techniques in Egypt.

\section{DESIGN, IMPLEMENTATION, AND EXPERIMENTAL RESULTS OF THE CVR}

The CVR is the most preferred receiver for the implementation of RWR. This is because the CVR has a simple structure, a low cost, an almost $100 \%$ probability of intercept (POI), a wide input bandwidth, a low weight, and a small size. It can measure the following threat signal parameters: pulse width (PW), pulse repetition frequency (PRF), pulse amplitude (PA), angle of arrival $(\mathrm{AOA})$, and frequency band instantaneously. The block diagram of the crystal video detector is shown in Fig.1. The main element in the CVR is the diode detector which is the nonlinear element necessary for extracting the useful information from the received signals. A Si-beam lead Schottky diode of type HP 5082-2207 was used in the design. The outline of the diode is shown in Fig.2 and the equivalent circuit is shown in Fig.3. The CVR is designed to operate from 1.5 to $4 \mathrm{GHz}$. The most important problem in the design is how to match the impedance of the diode detector through this wide frequency range to the standard characteristic impedance of microwave transmission lines which is $50 \Omega$. In order to perform this matching, it was necessary to use three matching elements as shown in Fig. 4. The first matching element is a series line that is used to rotate the diode impedances on the Smith chart until the resulting admittance has the same susceptance at the band edges. The second matching element is a short-circuited stub, designed such that the net susceptance at the band edges approaches zero. The third element is a series line, designed to equalize the VSWR through the band. The matching elements are designed using the microstrip technology. The equations and formulas used in the design are given elsewhere [3-6]. Fig.5 illustrates clearly the effect of the matching elements on the input VSWR of the CVR. The layout of the implemented CVR negative mask is shown in Fig.6. It was fabricated and mounted at the Industrial Design Development Center in Dar El-Salaam, Cairo, Egypt. The implemented crystal video detector is shown in Fig.7. The experimental setup illustrated in Fig. 8 was used to measure the output voltage as a function of the input power. The results at the centeral frequency $(3 \mathrm{GHz})$ are given in Fig.9. Fig.10 shows the dependence of the output voltage versus the operating frequency for different values of the input power level.

The measurement of the input, VSWR necessitated the use of the more complicated setup shown in Fig.11. The results obtained are presented in Fig.12. It is seen that the VSWR is very adequate for detector applications as it is indicated elsewhere [3].

In order to provide the CVR with a frequency measurement capability, the crystal video detector must be preceded by a frequency multiplexer like the one shown in Fig.13. The blocks labelled $f i$ where $i=1,2, \ldots n$ are bandpass filters. An interdigital bandpass filter was designed at the center frequency of $3 \mathrm{GHz}$. The mask layout of this filter is shown in Fig.14. 


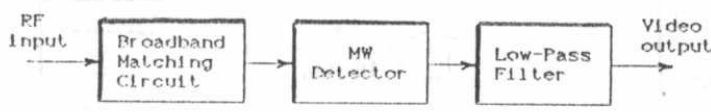

Fig.1 Block diagram of a CVR

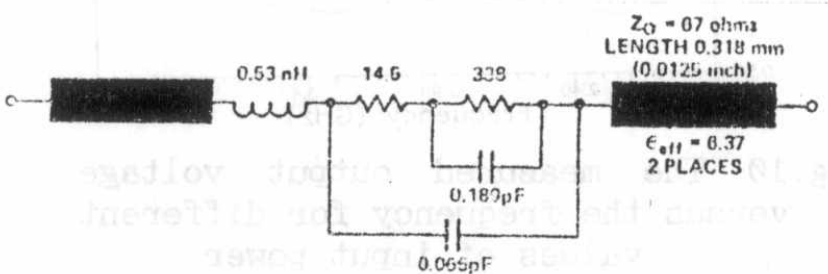

Fig.3 Equivalent circuit of the diode

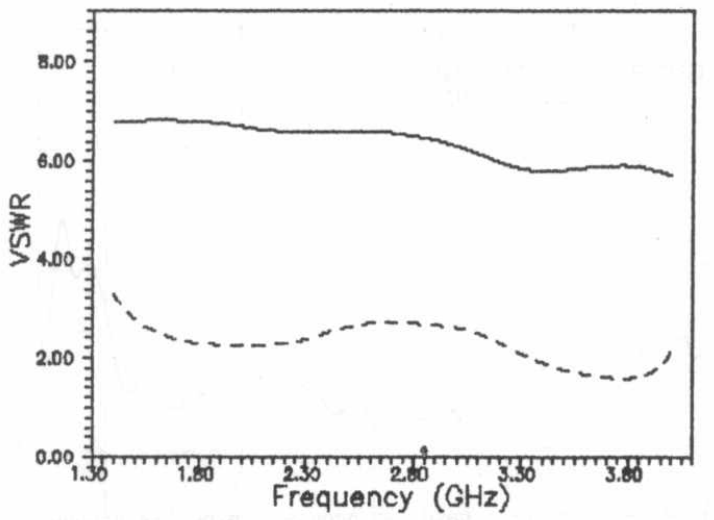

Fig.5 The input VSWR without matching ( - ), and with matching ( - )

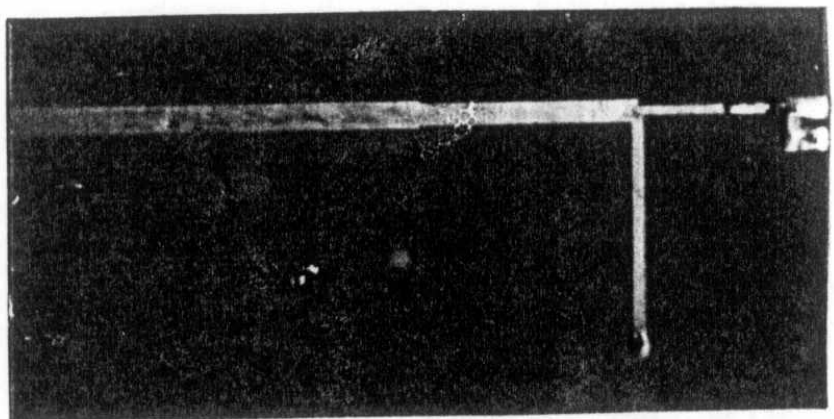

Fig. 7 The implemented CVR
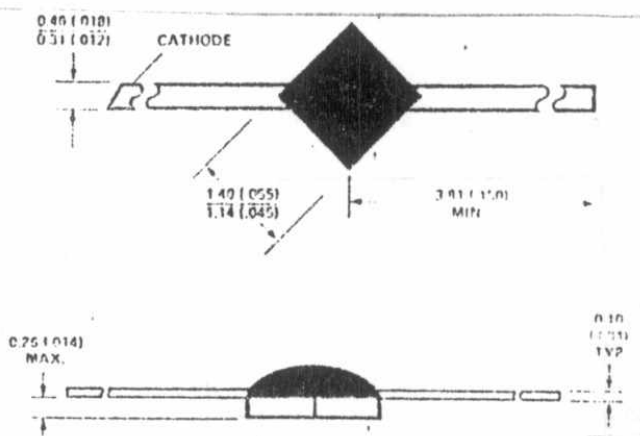

Fig.2 Outline of the diode HP 5022-2207

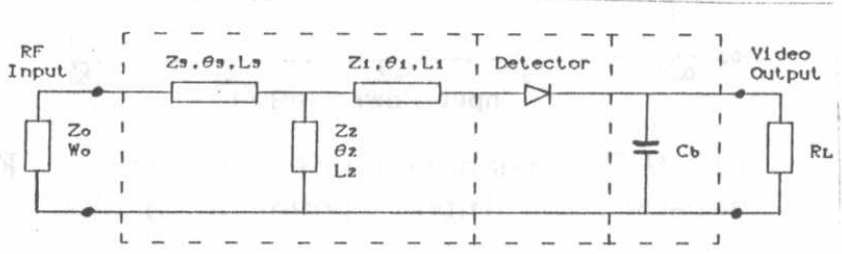

Fig. 4 A CVR with the matching elements

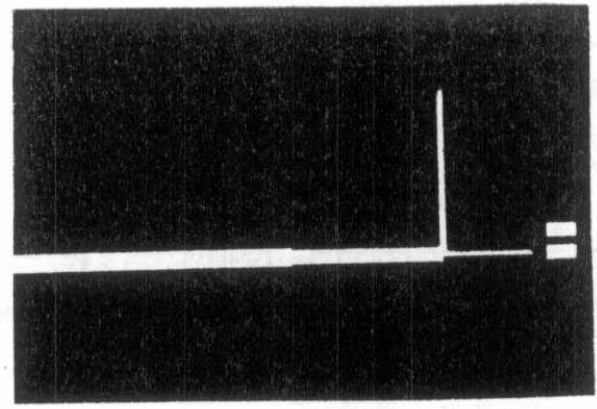

Fig.6 The implemented CVR negative mask

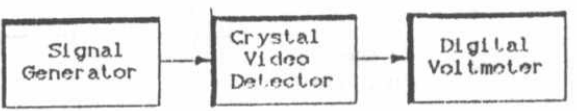

Fig. 8 Block diagram of the experimental setup used in testing CVR 


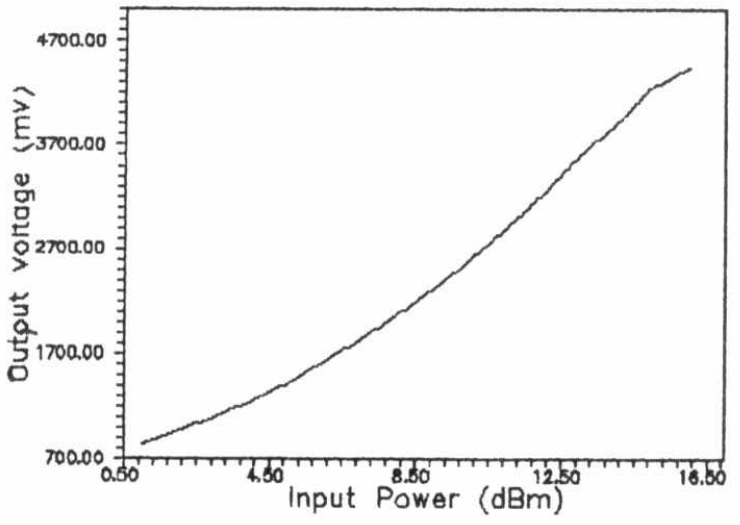

Fig.9 The measured output voltage versus the input power at $3 \mathrm{GHz}$

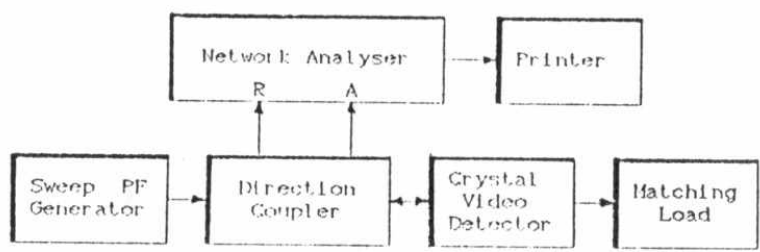

Fig.11 Block diagram of the experimental setup used to measure the input VSWR of the CVR

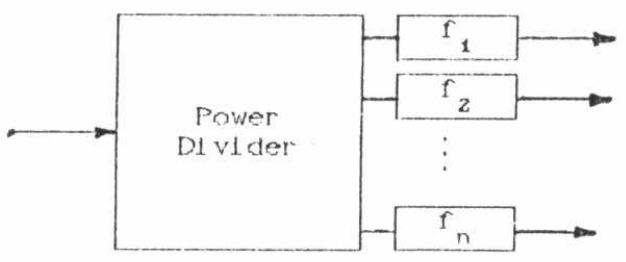

Fig. 13 Block diagram of the frequency multiplexer

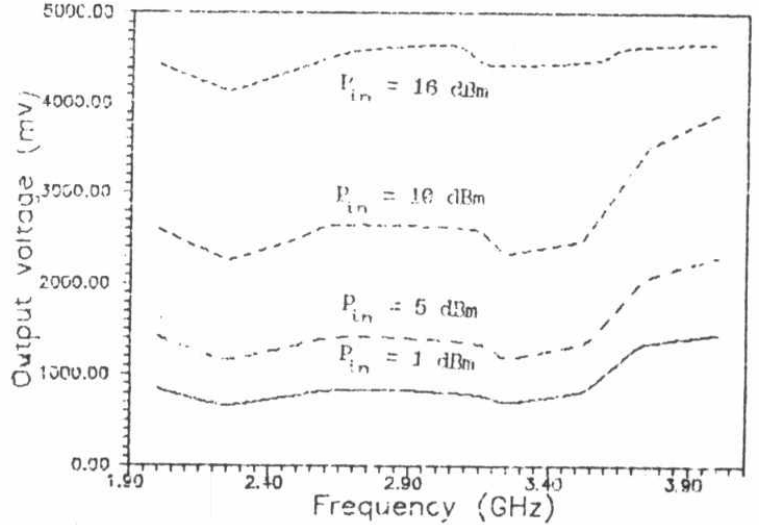

Fig.10 The measured output voltage versus the frequency for different values of input power

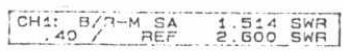

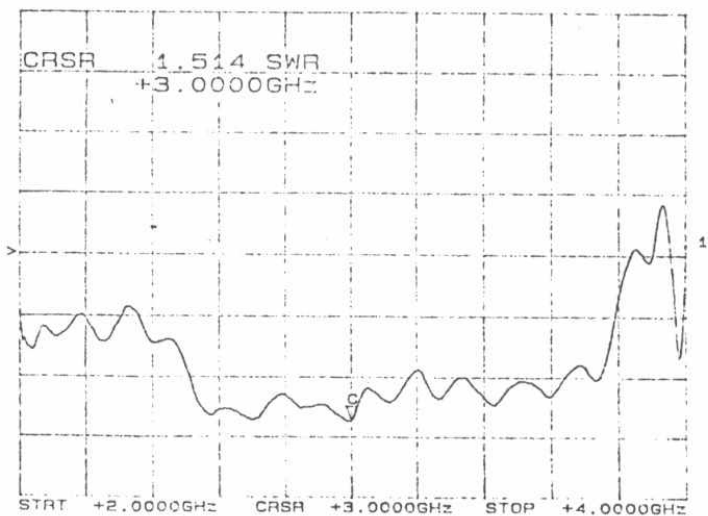

Fig.12 The measured input VSWR of the CVR

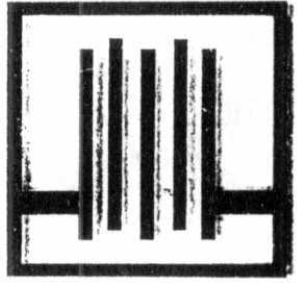

Fig.14 The mask layout of the interdigital filter 


\section{DESIGN, IMPLEMENTATION, AND EXPERIMENTAL RESULTS OF THE IFM RECEIVER}

In RWRs, the frequency is the main important parameter used for sorting and deinterleaving radar signals in dense signal environments. At present, IFM receiver is the most commonly used device for measuring frequency in RWRs system, because it has many good performance characteristics, such as a $100 \%$ POI, a good wide bandwidth, a high frequency measurement accuracy and resolution, an ability to detect fixed frequency, frequency agile, and chirped signals, a wide dynamic range, a good sensitivity, an instantaneous frequency analysis, and an amplitude independent analysis. Furthermore, it is compact in size and light in weight.

The basic block diagram of the IFM receiver is shown in Fig.15. The IFM receiver divides the input signal into two paths, one having a delay line of known length and the other is a direct path. The two signals are then applied to a phase correlator which provides four video voltages. These voltages are processed in the video and digital section to determine the phase angle $\theta$ that is proportional to the signal frequency. Hence, the signal frequency can be determined. The most critical components of the IFM receiver are the delay line and the phase correlator [7-8]. The delay line is designed and implemented as an integral part of the microstrip circuit comprising the phase correlator. If the required delay time is $\mathrm{T}_{\mathrm{d}}$, then the delay line length is given by :

$$
L=V T_{d}=T_{d} c / \sqrt{\varepsilon_{\text {fff }}},
$$

where $\mathrm{c}$ is the velocity of light, and $\varepsilon_{\text {eff }}$ is the effective relative dielectric constant.

Fig. 16 shows four possible configurations of the phase correlator. The main problem of all these configurations is the crossover between the RF main problem of all these con partially solved at the expense of the VSWR [9].
strips. This problem can be partion adopted solution to this problem is shown in Fig.17. The solution is based
Our a on using an appropriate combination of Lange couplers [3] and inphase Wilkinson power dividers [10] in order to avoid any intersection between the strips. The overall performance of the IFM receiver depends on the bandwidth of the used microwave components. These components are: $3 \mathrm{~dB}$ inphase power dividers, $3 \mathrm{~dB} 90$ degs. hybrids, broadband matching circui detectors. The $3 \mathrm{~dB}$ inphase power splitter can be realized using either $a$ Wilkinson power divider, a ring hybrid, or a modified ring hybrid. The Wilkinson power divider is the simplest one, but the modified ring hybrid has a better performance. The $3 \mathrm{~dB} 90$ degs. hybrid can be realized using either a branch line hybrid, a coupled line hybrid, or a Lange coupler. The branch line hybrid is the simplest one, but its usable bandwidth is limited to $10 \%$; the phase difference between output ports is frequency dependent, and its line impedances are sometimes not practically realizable. Multisection branch line coupler can improve the performance, but the circuit will be large. Coupled line hybrid has a good amplitude and phase performance, but the required space between the two lines is too small to fabricate accurately, also the output ports emerge on opposite sides. Using the Lange coupler is the solution to the problems encountered in both branch line coupler and the coupled line hybrid. This line has a good amplitude and phase performance, its out ports emerge on the same side, and it is practically realizable [11-12]. Hence, the Lange coupler is the most suitable $3 \mathrm{~dB} 90$ degs. hybrid for the phase correlator 


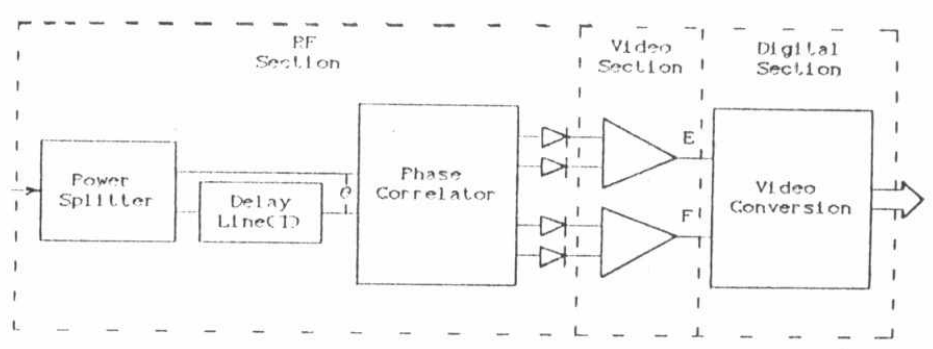

Fig.15 The block diagram of the IFM receiver
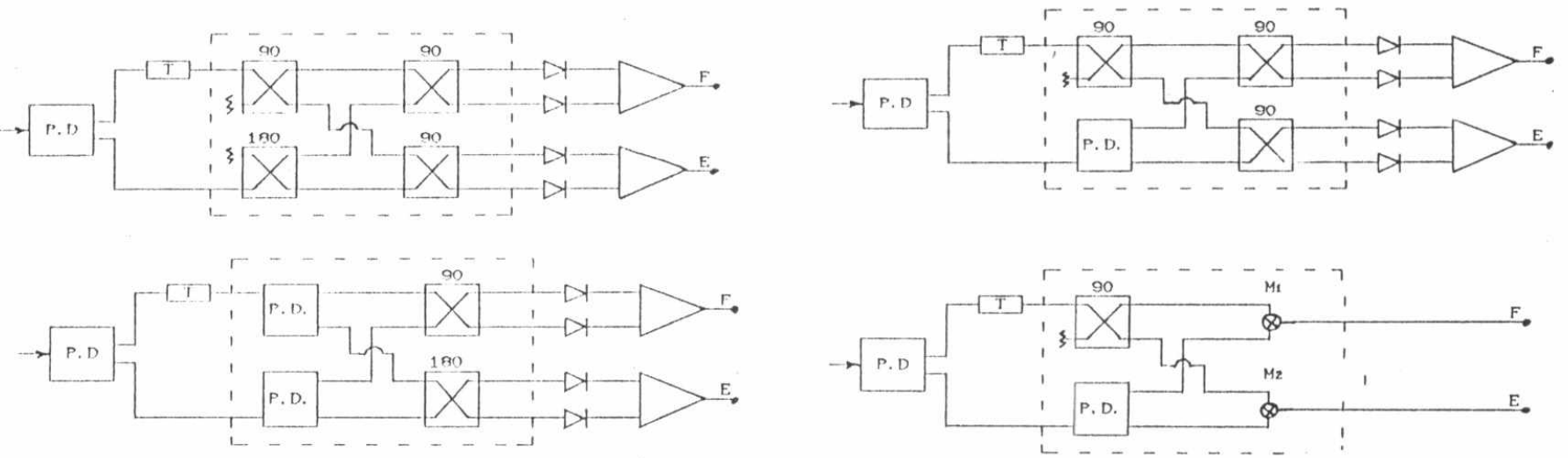

fig. 16 'I'he possible configurations of the phase correlator
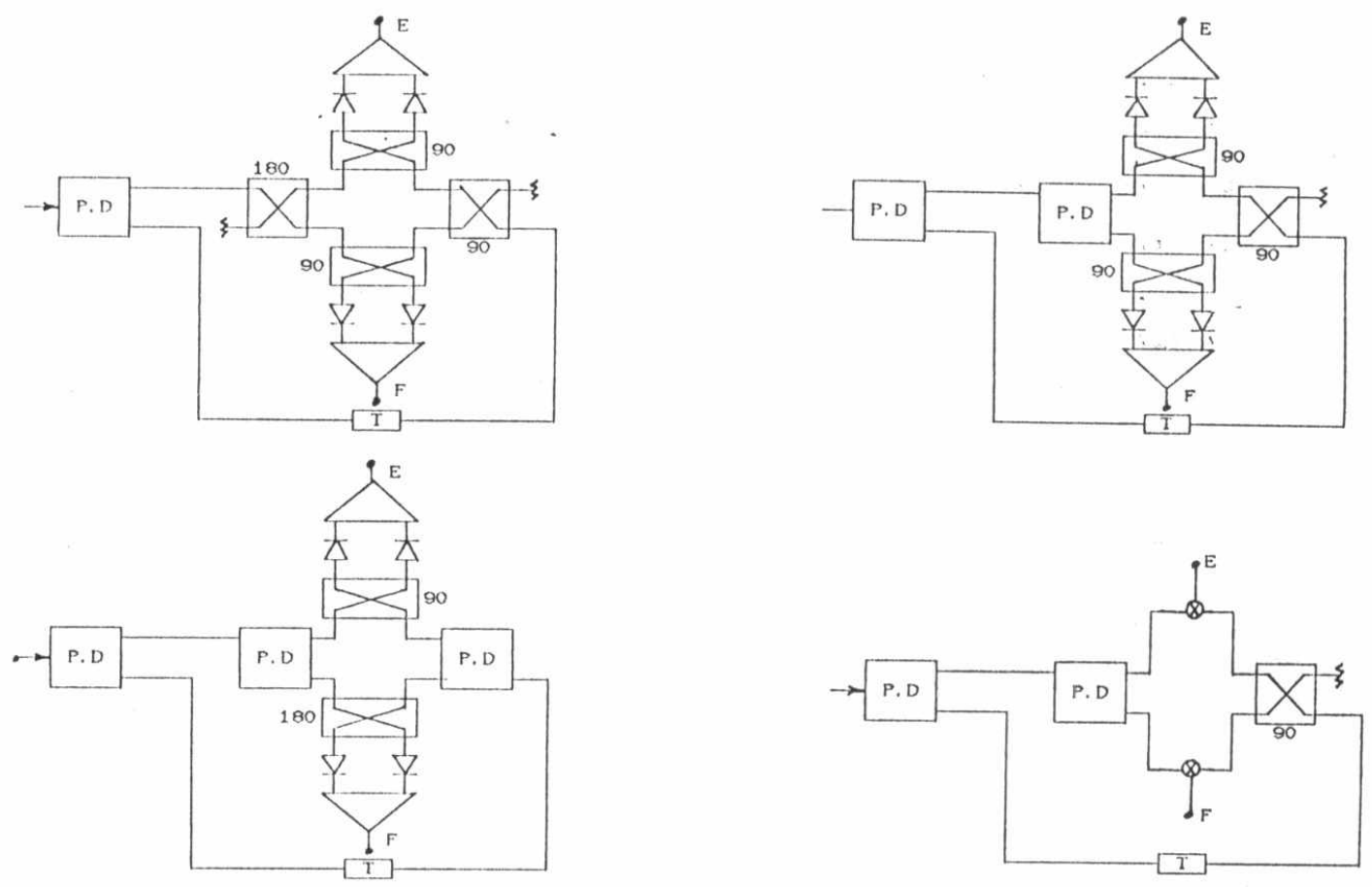

Fig.17 Modified phase correlator without strip intersection 
design. A Si-beam lead Schottky diode of type HP 5082-2207 was used in the design. In order to achieve matching between diode impedances and the hybrid, three matching elements were used as it is described in the previous section.

The following procedure is proposed to design the IFM receiver :

1. Select a suitable IFM receiver structure from Fig.17.

2. Select the microwave hybrids, couplers, and power dividers which cover the required frequency band, achieve good performance through the required frequency band, and can be realized according to the available technology.

3. Choose the microstrip substrate material which is suitable for designing the mentioned microwave components.

4. Calculate the delay line length which cover the required frequency band without ambiguity. If the required delay is short, the delay line can be implemented as an integral part of the microstrip. If the delay is long, the delay line can be implemented using a section of a coaxial line of low attenuation. In this case the delay line must be attached to the microstrip substrate.

5. From the microwave and RF designer's catalog select the diode detector which cover the required frequency band.

6. Calculate the diode impedances as a function of the operating frequency band.

7. Design the broadband matching circuits between the diodes and the transmission lines of standard characteristic impedance.

8. Design the low pass filter following the detectors to stop all the high frequency components.

9. Design the two stage differential video amplifiers.

10. Design the digital circuit for encoding the IFM receiver output.

The block diagram of the microwave part of the IFM receiver is shown in Fig.18. Computer programs were developed to calculate the the impedances, and lengths of the the three-element matching circuits. The locus of the resultant admittance on Smith chart is shown in Fig.19. The designed matching elements are shown in Fig. 20. The layout of the implemented IFM receiver mask is shown in Fig.21. The fabrication and mounting process were carried out in Banha Company for Electronic Industries, Kalyobia, Egypt. The photograph of the implemented microwave part of IFM receiver with substrate holder, BNC connectors, and launcher is shown in Fig.22.

The designed IFM receiver was tested in the Main Radar Workshop No.1, Cairo, Bgypt. The measuring set up is shown in Fig.23. The signal generator (model HP-8350A) is used for feeding input RF signal from 2 to $4 \mathrm{GHz}$. The 20 $\mathrm{dB}$ attenuator was used to attenuate the output RF. The $3 \mathrm{~dB}$ power splitter was used to divide the input RF power into two paths, one to the IFM receiver, and the other to the powermeter (model HP-8481). The oscilloscope (model-HP-1740A) with $1 M \Omega$ input resistance, which corresponds to diode detector load resistance was used to measure the four output dc voltages from the IFM receiver at different frequencies. The two bipolar input voltages versus the input frequency are shown in Fig.24. During the measurement, the RF input power was kept constant, and the RF input frequency was varied from 2 to $4 \mathrm{GHz}$

The output voltage is almost a sinusoidal function of the operating frequency. The deviation from the expected sinusoidal dependence is attributed to the bandwidth limitations of the used components and the fabrication tolerances. However, it must be noted that most practical IFM receivers use correction circuits to compensate this deviation. 


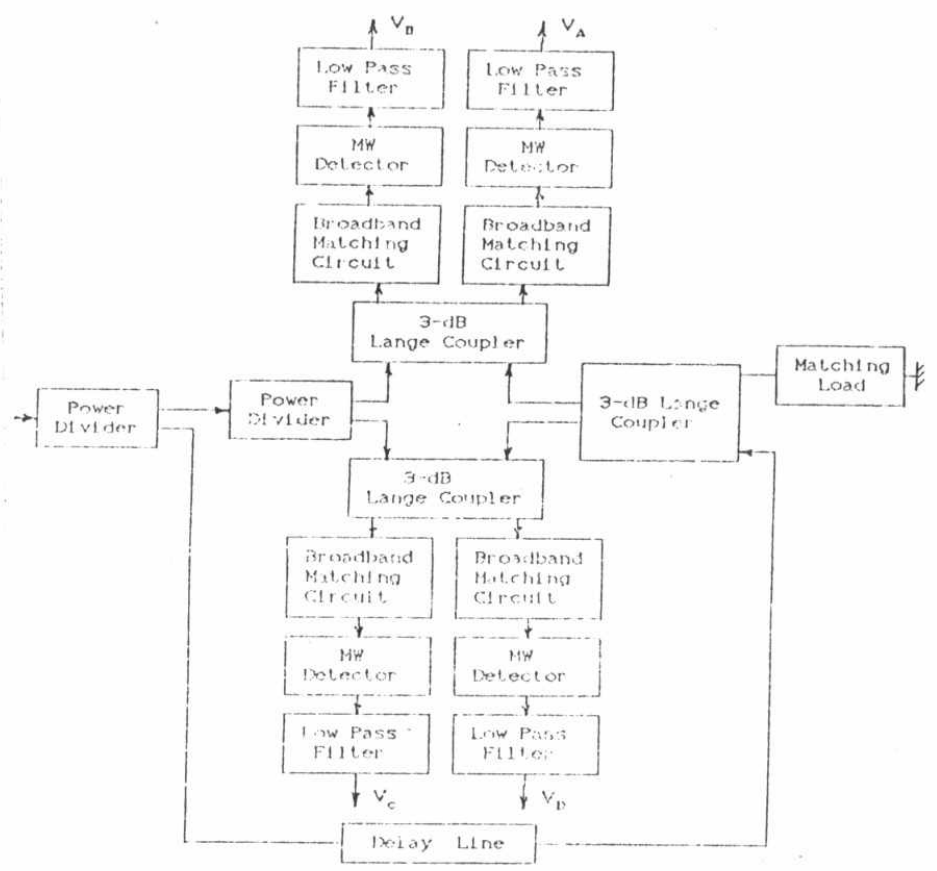

Fig.18 The block diagram of the microwave part of the IHM receiver

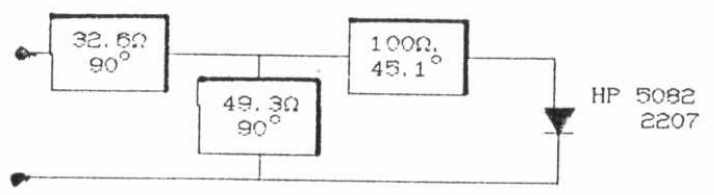

Fig.20 Impedances and electrical lengths of matching elements

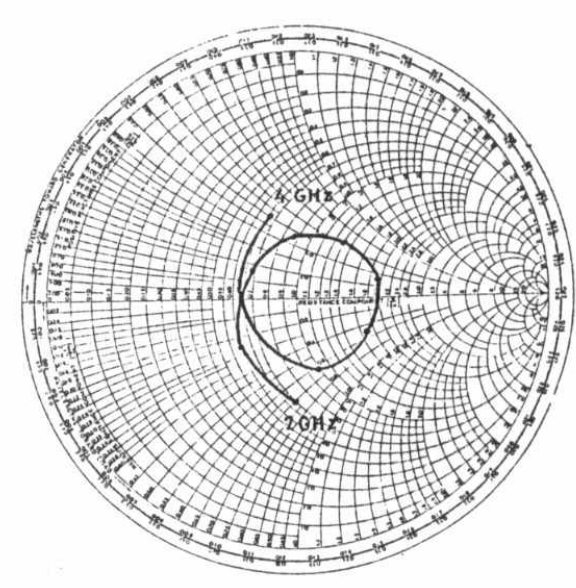

Fig.19 Impedances of diode with three e]ement matching circuit

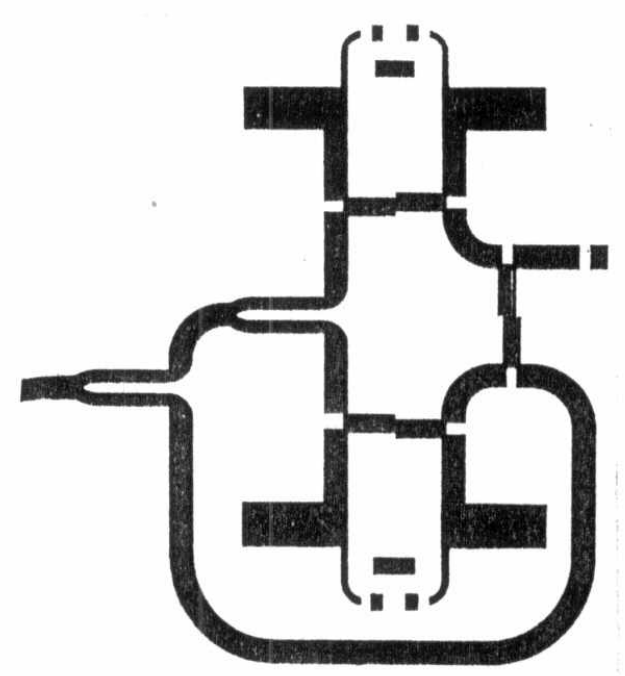

Fig. 21. The implemented IFM mask receiver 


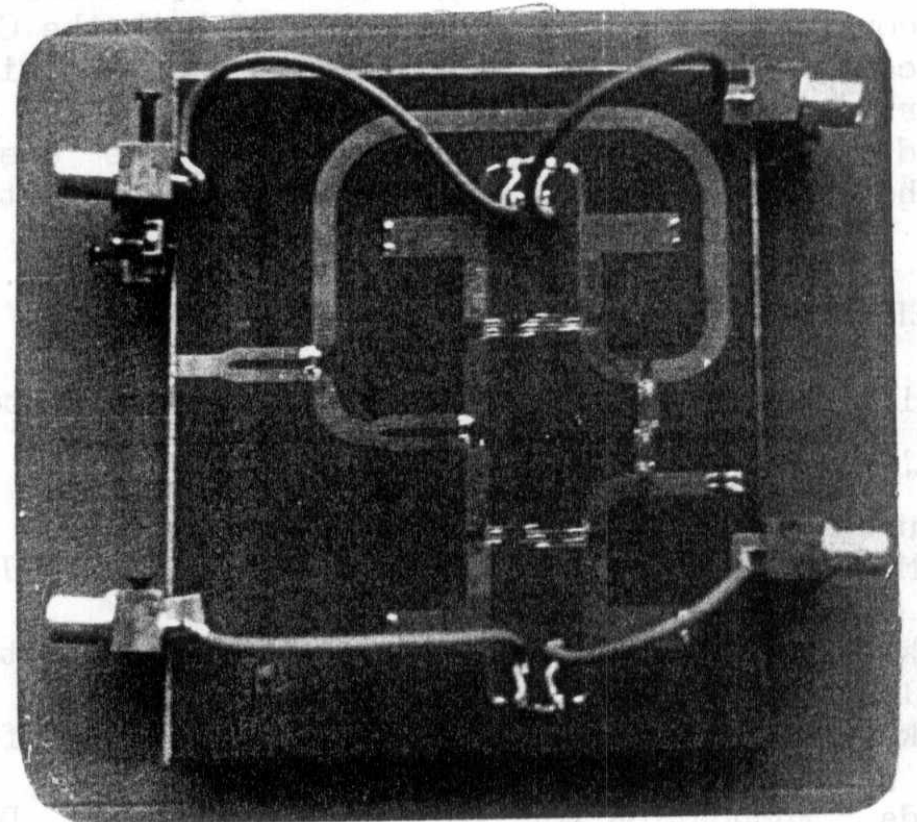

Fig.22 The implemented microwave part of the IFM receiver

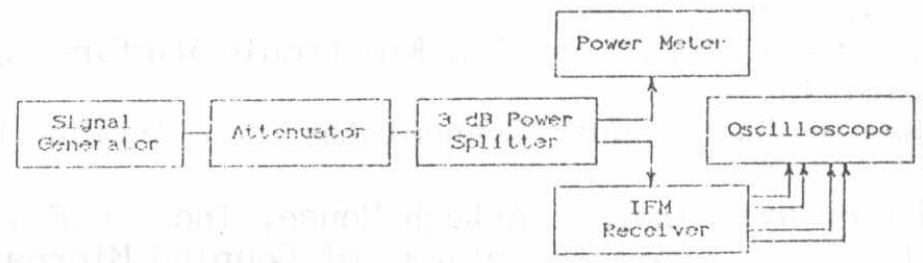

Fig. 23 The measuring setup for testing the IFM receiver

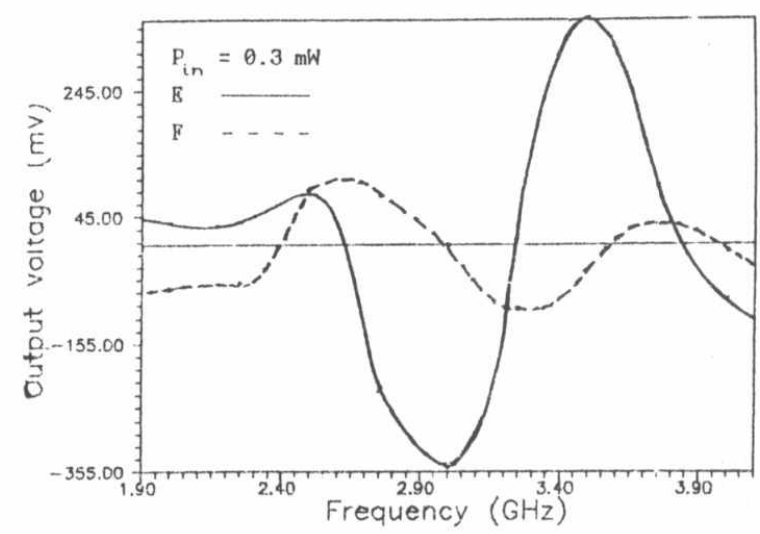

Fig.24 Measured output voltage versus frequency 


\section{YI CONCLUSION}

In this paper we have presented a successful design and implementation of the critical components of radar warning receiver, operating in the S-band. These components constitute the microwave parts of both the CVR and the IFM receivers. These components were designed and implemented using the recently developed microstrip technology. New design concepts and a design procedure have been proposed and adopted for the IFM receiver. The designed components were tested and the measured output parameters were satisfactory.

\section{Y REFERENCES}

[1] Filippo Neri, " Introduction to Electronic Defense Systems ", Artech House, Inc., 1991.

[2] D. White, "Advances in Radar Warning Receivers", International Countermeasures Handbook, 1983.

[3] I. Bahl, "Microwave Solid State Circuit Design ", John wiley and sons, Inc., U.S. 1988.

[4] Hewlett Packard " Schottky Barrier Diode Video Detectors ", Hewlett Packard Application Note 923.

[5] Hewlett Packard ," Impedance Matching Techniques for Mixers and Detectors", Hewlett Packard Application Note 963.

[6] 'T. C. Edwards," Foundation for Microstrip Circuits Design ", John wiley and sons, 1981.

[7] P. Fast," Design Technigues and Performance of Digital IFM ", IEB proc., Vol.129, Pt, F, No.3, June 1982.

[8] J. P. Y. Lee," Detection of Complex and Simultaneous Signals Using an Instantaneous Frequency Measurement Receiver ", IEE Proc., Vol.132, Pt. F, No. 4, July 1985.

[9] James B.T.,"Microwave Receivers with Electronic Warfare Applications", Contervill, Ohio, 1986.

[10] E. D. Ost.," Solid State Radar Transmitter ", Artech House, Inc., 1985.

[11] S. A. Mass," Microwave Mixers", Artech House, Inc., U.S., 1986.

[12] G. R. and I. J. Bahl," Characteristics of Coupled Microstrip" IEEE Trans. Microwave Theory Tech., Vol. MTT'-27, July 1979, pp. 700-705. 